\title{
Underwater acoustic channel properties in the Gulf of Naples and their effects on digital data transmission
}

\author{
Tony Alfredo Stabile $\left({ }^{1}\right)$, Aldo Zollo $\left({ }^{1}\right)$, Maurizio Vassallo $\left({ }^{1}\right)$ and Giovanni Iannaccone $\left({ }^{2}\right)$ \\ (1) Dipartimento di Scienze Fisiche, Università degli Studi di Napoli Federico II, Napoli, Italy \\ ${ }^{(2)}$ Istituto Nazionale di Geofisica e Vulcanologia, Osservatorio Vesuviano, Napoli, Italy
}

\begin{abstract}
In this paper we studied the physical properties of the Gulf of Naples (Southern Italy) for its use as a communication channel for the acoustic transmission of digital data acquired by seismic instruments on the seafloor to a moored buoy. The acoustic link will be assured by high frequency acoustic modems operating with a central frequency of $100 \mathrm{kHz}$ and a band pass of $10 \mathrm{kHz}$. Since the maximum depth of the sea is about $300 \mathrm{~m}$ and the planned horizontal distance between the seismic instruments and the buoy is $2 \mathrm{~km}$, the acoustic data transmission shall be near horizontal. In this study the signal-to-noise ratio is plotted against depth and distance from the source, thus defining the limit after which the transmitted information becomes unreliable. Using ray-theory, we compute the amplitudes of a transmitted signal at a grid of $21 \times 12$ receivers to calculate the transmission loss at each receiver. The signal-to-noise ratio is finally computed for each receiver knowing also the transmitter source level and the acoustic noise level in the Gulf of Naples. The results show that the multipath effects predominate over the effects produced by the sound velocity gradient in the sea in the summer period. In the case of omnidirectional transmitters with a Source Level (SL) of $165 \mathrm{~dB}$ and a bit rate of $2.4 \mathrm{kbit} / \mathrm{s}$, the results also show that distances of 1400-1600 $\mathrm{m}$ can be reached throughout the year for transmitter-receiver connections below $50 \mathrm{~m}$ depth in the underwater acoustic channel.
\end{abstract}

Key words underwater acoustics - signal-to-noise ratio - shallow water - high frequency - Gulf of Naples

\section{Introduction}

Over the last ten years, there has been a rapid extension in the use of digital underwater acoustic communication from mainly military purposes to those commercial, to satisfy the growing needs for underwater acoustic wireless communication systems in real time, and to over-

Mailing address: Dr. Tony Alfredo Stabile, Dipartimento di Scienze Fisiche, Università degli Studi di Napoli Federico II, Complesso Universitario di Monte Sant'Angelo, Via Cintia, 80126 Napoli, Italy; e-mail: stabile@na.infn.it see the submerged instrumentation without the direct intervention of man.

Underwater acoustic wireless sensor networks are an emerging topic of research and are envisioned to enable applications on the surveillance and monitoring of sea areas (Adams et al., 2000; Sozer et al., 2000; Akyildiz et al., 2005; Benson et al., 2006; Jaffe et al., 2006). There is, in fact, significant interest in monitoring aquatic environments for scientific, environmental, commercial, safety and military reasons such as detect tectonic movements, incoming tsunamis, water pollution, global warming, distributed tactical surveillance, mine reconnaissance, and many other facts that are bound to affect our lives (Badia et al., 2006; Pompili et al., 2006).

The main problem of underwater acoustic communication is the complexity of the marine 
acoustic channel: background noise from the environment and from human activities; unevenness in the water caused by the presence of solid and gaseous particles; the presence of spatial and temporal sound velocity gradients that can arise from salinity, pressure and temperature variations; multipath effects caused by multiple reflections on the sea surface and bottom; reverberations caused by the agitation of the sea and the topography of the sea bottom; and marine currents. In particular, with a digital communication system, the multipath that occurs with the propagation of signals results in intersymbol interference (ISI), which limits the capacity of the underwater acoustic communication channel. All of these phenomena are magnified in the situation of acoustic transmission in shallow water with respect to transmission in deep water, due to the depth of the sea and the vicinity to the coast (Cazzolato et al., 2001; Smith et al., 2003), so the shallow water acoustic communication channel is characterized by strong signal degradation.

The use of high frequencies in acoustic communication improves the velocity of digital transmission of data, but also causes a greater attenuation of the signal (Thorp, 1967; Fisher and Simmons, 1977), with the consequent decrease in the range of distance, i.e. in the ability to transmit data over distance.

Acoustic communication has been progressively improving over the years, both in terms of the distances and the data transmission capacity, due to the development of new techniques of signal modulation. In more recent years, the feasibility of modulation techniques for underwater communication and telemetry have been demonstrated (Baggeroer, 1984; Catipovic, 1990; Coates, 1993; Stojanovic et al., 1993, 1994, 1995; Stojanovic, 1996), minimizing the ISI to improve the capacity of the transmission channel. Internationally, the systems of acoustic links that are now most advanced use frequencies around 20 $\mathrm{kHz}$ (ultrasound), reaching bit rates of $10 \mathrm{kbit} / \mathrm{s}$ (Stojanovic, 2004).

To be able to complete any underwater acoustic communication system, it is necessary to know the physical properties of the transmission channel to be able to understand what the limits on signal transmission are and how its complexi- ty can influence the quality of the information transmission.

In the Gulf of Naples (Southern Italy) a feasibility test is ongoing which provides for the installation of seismic instruments on the seafloor to extent offshore the geophysical monitoring of the Neapolitan volcanoes, Mt. Vesuvius and Campi Flegrei caldera. The seismic data loggers will be acoustically linked to a moored buoy using a two-way communication modem, then a radio apparatus on the buoy will telemeter data on land.

Here, we studied the physical properties of the water layer of the Gulf of Naples for its use as an acoustic communication channel for highfrequency digital transmissions (around 100 $\mathrm{kHz}$ ), and analyzed the influence of the seasonal variations in its physical parameters on underwater transmission and the limits that the channel forces upon this communication. For this purpose we are interested to understand the effects produced by the underwater acoustic channel on a high frequency digital transmission to choose the best configuration of the communication system. We do not take into account the effects of bubble scattering and absorption because the layer that contains bubbles is near the surface (Preisig, 2006) and, as our models use wind speeds less than $6 \mathrm{~m} / \mathrm{s}$, bubbles cannot significantly attenuate propagating signals in this layer (Dahl, 2004). We also consider the sea as a steady propagation environment with smooth sea surface and seafloor during the transmission of data.

The first section provides a description of the physical properties of the Gulf of Naples as a one-dimensional (1D) communication channel in shallow water, considering that the average depth of this water layer is less than $300 \mathrm{~m}$. In the second section, the potential noise sources are described, and the Noise Levels (NL) expected at the $100 \mathrm{kHz}$ frequency are calculated. In the third section, we proceed to the simulation of underwater acoustic propagation, describing the calculation method carried out to determine the Signal-to-Noise Ratio (SNR). Finally, in the fourth section, the results we obtained for a standard piece of apparatus for digital acoustic transmission of information are reported, evaluating the limits of high frequency acoustic transmis- 
sion in the Gulf of Naples, starting from the SNR calculated from the sea surface to the bottom, and up to a distance of $2 \mathrm{~km}$.

\section{One-dimensional velocity model of the Gulf of Naples}

The Gulf of Naples is located along the south-western coast of Italy, between $40^{\circ} 35^{\prime}$ and $40^{\circ} 50^{\prime}$ latitude north, and between $14^{\circ} 00^{\prime}$ and $14^{\circ} 30^{\prime}$ longitude east. The depth of the sea in the area of interest is not deeper than $300 \mathrm{~m}$, except for the northern part of the Dohrn canyon (fig. 1), in which the sea depth reaches $400 \mathrm{~m}$. Since the Gulf of Naples is not deeper than $300 \mathrm{~m}$, it can be considered a shallow water acoustic communication channel. This im- plies a greater complexity on the sound propagation because in shallow water the surface, volume, and bottom properties are all important. Moreover the seasonal variation in soundspeed structure is significant with winter condition being nearly isospeed (Jensen et al., 2000).

The essential characteristic of sea water is, in fact, the velocity at which the sound can be propagated through it. The square of the velocity of the sound depends on the ratio between the density and the bulk modulus of the propagation medium. In particular, in the sea the density is a function of the pressure, temperature and salinity of the sea, while the bulk modulus is a function of the pressure and temperature of the sea. For this reason, the propagation velocity of acoustic energy in the sea can be described as a function of three physical parameters: pressure,

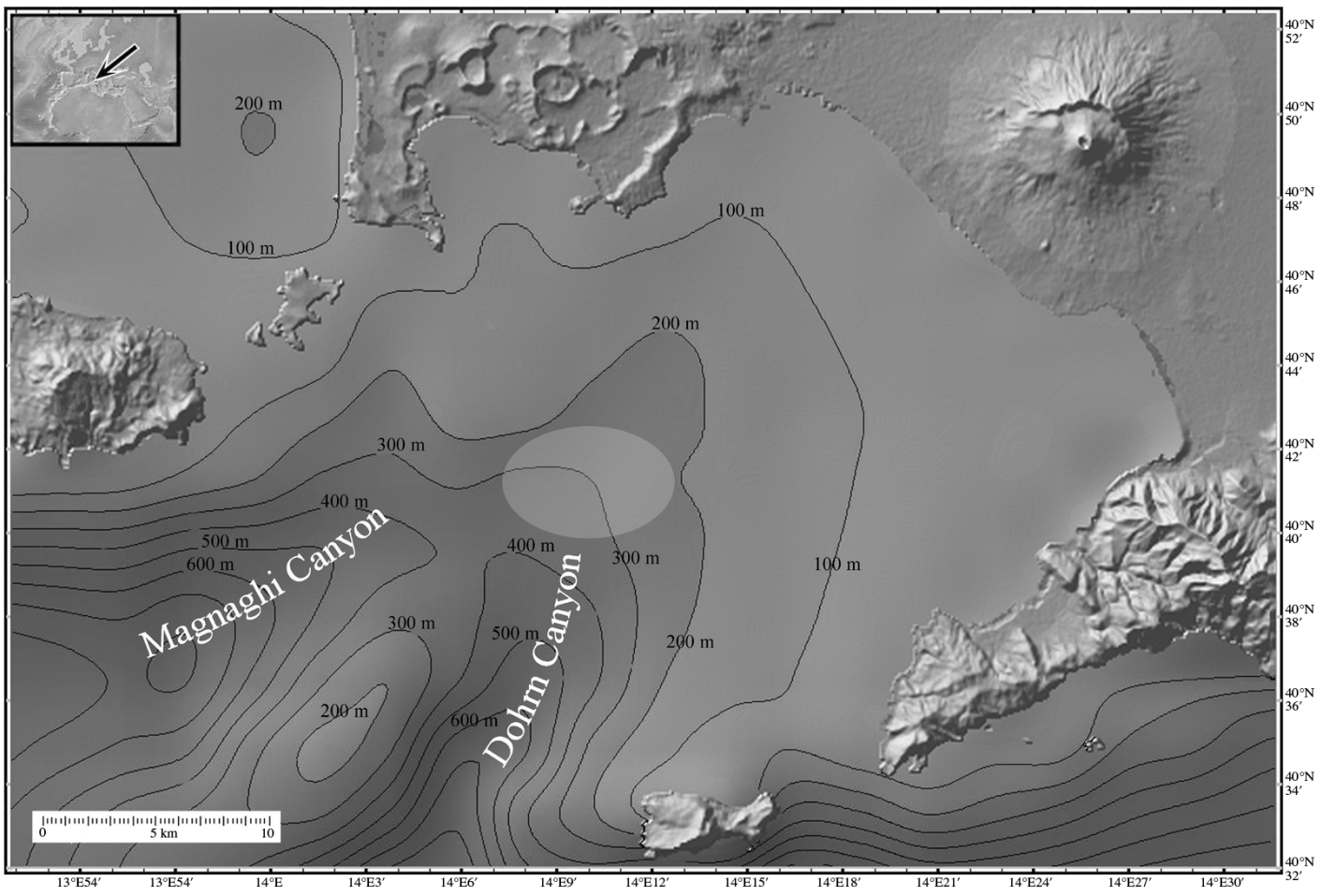

Fig. 1. Bathymetric and topographic image of the Gulf of Naples. A bright ellipse indicates the area of interest for our studies, where the researchers of the Anton Dohrn Zoological Station carried out measurements of the physical properties of the seawater in the period from February 2002 to February 2003. 
temperature and salinity (Jensen et al., 2000). It is not only necessary to know the mean values of these three physical parameters for the sea, but it is also important to know their spatial and temporal variabilities, as these variations significantly modify the propagation velocity of the acoustic waves in the sea. Over the years, various formulae have been suggested that link the sound velocity in the sea to the three parameters of pressure, temperature and salinity (Kinsler et al., 2000; Mackenzie, 1981; Coppens, 1981), and in particular, the UNESCO algorithm (Chen and Millero, 1977; Wong and Zhu, 1995) and the equation of Del Grosso (Del Grosso, 1974) are commonly used.

If the sea is considered to be a resting fluid with a constant density $\rho$, the pressure is solely a function of the depth. There are also some very accurate, empirical relationships between sea depth and pressure. The sea temperature and salinity, however, depend upon the ambient conditions of the location, such as the solar irradiation, evaporation, the atmospheric conditions, the water circulation, the wind, the presence of rivers, and the geographical position.

The characterization of the Gulf of Naples starts, therefore, from the knowledge of the values and variabilities of these parameters in space and time. For this, the temperature and salinity data were analyzed from measurements carried out by the researchers of the Anton Dohrn Zoological Station (Ribera, personal communication, 2005) in the Gulf of Naples from the sea surface to a depth of $300 \mathrm{~m}$, in the period from February 2002 to February 2003. The sound velocity data, calculated by the same with the equation of Del Grosso (Del Grosso, 1974) from these salinity and temperature measurements, were analyzed.

Figure 2 shows the salinity profiles of the sea between the surface and the bottom at $300 \mathrm{~m}$ in depth, with a sampling rate of $0.1 \mathrm{~m}$, across 15 different periods of the year. The salinity went through small variations throughout the year, with a value of about $38 \mathrm{ppm}$. It can be seen from fig. 2 that there was an increase in the salinity with depth of about $0.5 \mathrm{ppm}$, from the surface to the bottom, and also rapid oscillations in the salinity values in the first $100 \mathrm{~m}$ in the non-winter periods due to surface marine currents, where at some depths the salinity can change of about 2 ppm from the mean value. In the winter periods, the strong agitation of the sea causes a mixing of the water down to great depth, resulting in stability of the salinity values.

Figure 3 shows the temperature profiles measured at the same time as the salinity at the same depths. The temperature varies with time, and on large spatial scales, in concomitance with the daily and seasonal variations of solar irradiation; fine spatio-temporal variations are determined locally by turbulent movements of the seawater. The variability in the temperature of the sea depends on the variability of the thermal content of the water as a consequence of the heat transfer between the surface and the adjacent atmosphere (De Dominicis Rotondi, 1995). From fig. 3 it can be seen that at the surface the higher temperatures are reached in the summer months, with maximum values of $27^{\circ} \mathrm{C}$ in August, while the lowest temperatures are reached in the winter months, with minimum values of $13^{\circ} \mathrm{C}$ in February; in the deepest water, the mean temperature is lower and the temperature variations between the summer and winter seasons are greatly reduced. In summer, water mixings hardly occur and results in a large negative temperature gradient in the first $75 \mathrm{~m}$ of depth, where the temperature can drop from $27^{\circ} \mathrm{C}$ at the surface to $14^{\circ} \mathrm{C}$ at $75 \mathrm{~m}$ in depth. In autumn and winter, the mixing of the water is very intense, which generates isothermic surface layers that reach down to a depth of $50 \mathrm{~m}$ in October to $160 \mathrm{~m}$ in February. For depths greater than $160 \mathrm{~m}$ the mean temperature is $14^{\circ} \mathrm{C}$ and its seasonal variation rarely reaches $0.1^{\circ} \mathrm{C}$; at this depth the temperature is no longer influenced by the outside meteorological conditions.

Figure 4 shows the sound velocity profiles calculated with the equation of Del Grosso (Del Grosso, 1974), starting from the salinity and temperature data. It can be inferred from this figure that the highest variations in the sound velocity are at the surface, and are between $1508 \mathrm{~m} / \mathrm{s}$ and $1541 \mathrm{~m} / \mathrm{s}$, equivalent to a percent variation of $\pm 1 \%$ of the mean velocity value. At increasing depths, the seasonal variations decrease until they become lesser than $5 \mathrm{~m} / \mathrm{s}$ below $100 \mathrm{~m}$ and negligible below $160 \mathrm{~m}$. A comparison between the sound velocity profiles with those of temperature reveal an overall correlation at all depths for all the periods of the year, except for a positive 
linear trend followed by the sound velocity profiles for increasing depths, caused by a rise in pressure with growing depth. From $160 \mathrm{~m}$ to 300 $\mathrm{m}$ in depth, where the temperature variations are negligible, the velocity only depends upon the pressure variations, increasing by around $2 \mathrm{~m} / \mathrm{s}$ The comparison between the salinity and sound velocity profiles shows that the rapid salinity oscillations in the hottest months, and for depths less than $100 \mathrm{~m}$, do not correspond to apprecia-
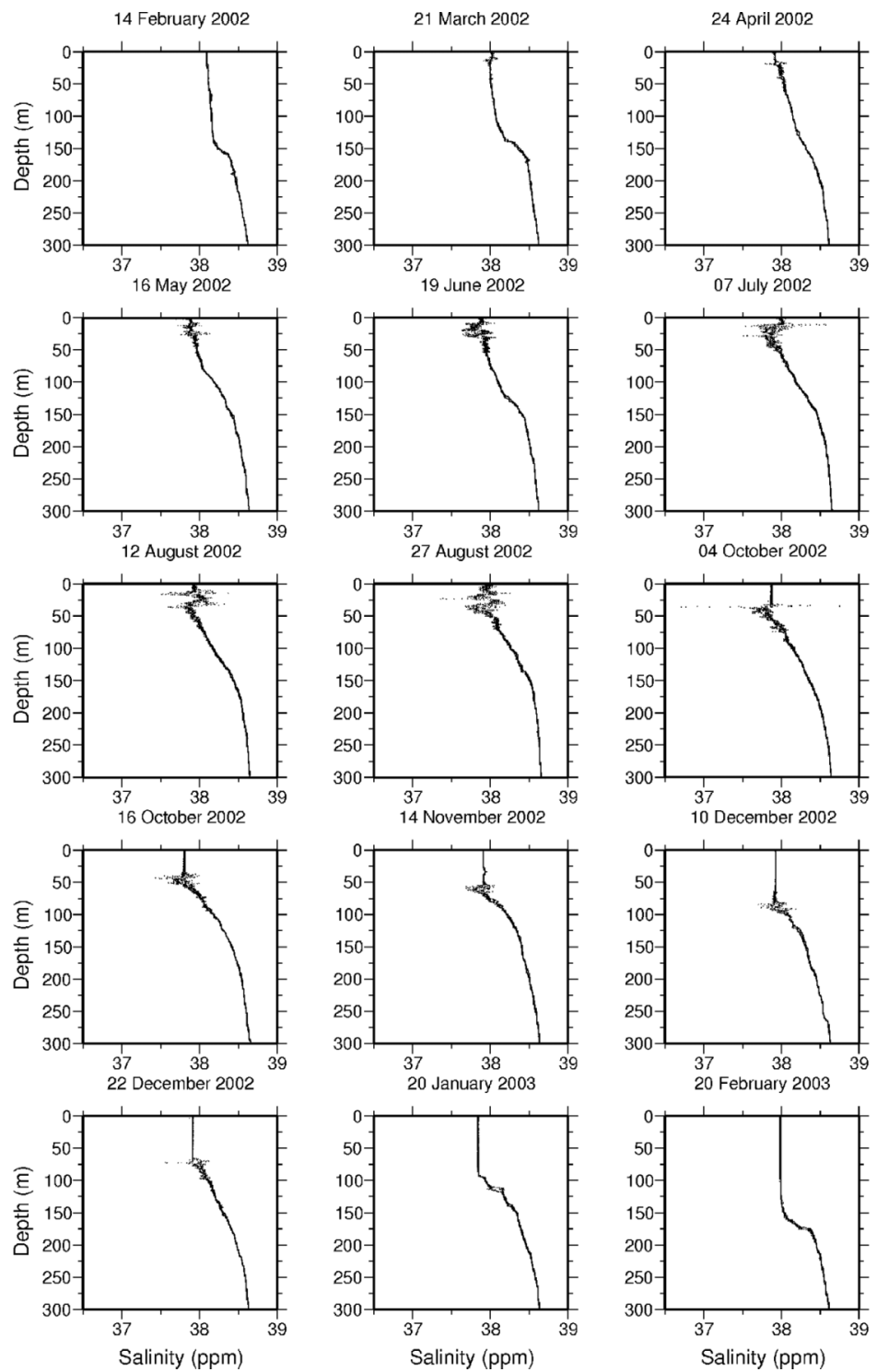

Fig. 2. Salinity profiles in the Gulf of Naples from the sea surface to the bottom, at $300 \mathrm{~m}$ in depth, measured across 15 different periods between February 2002 and February 2003. 

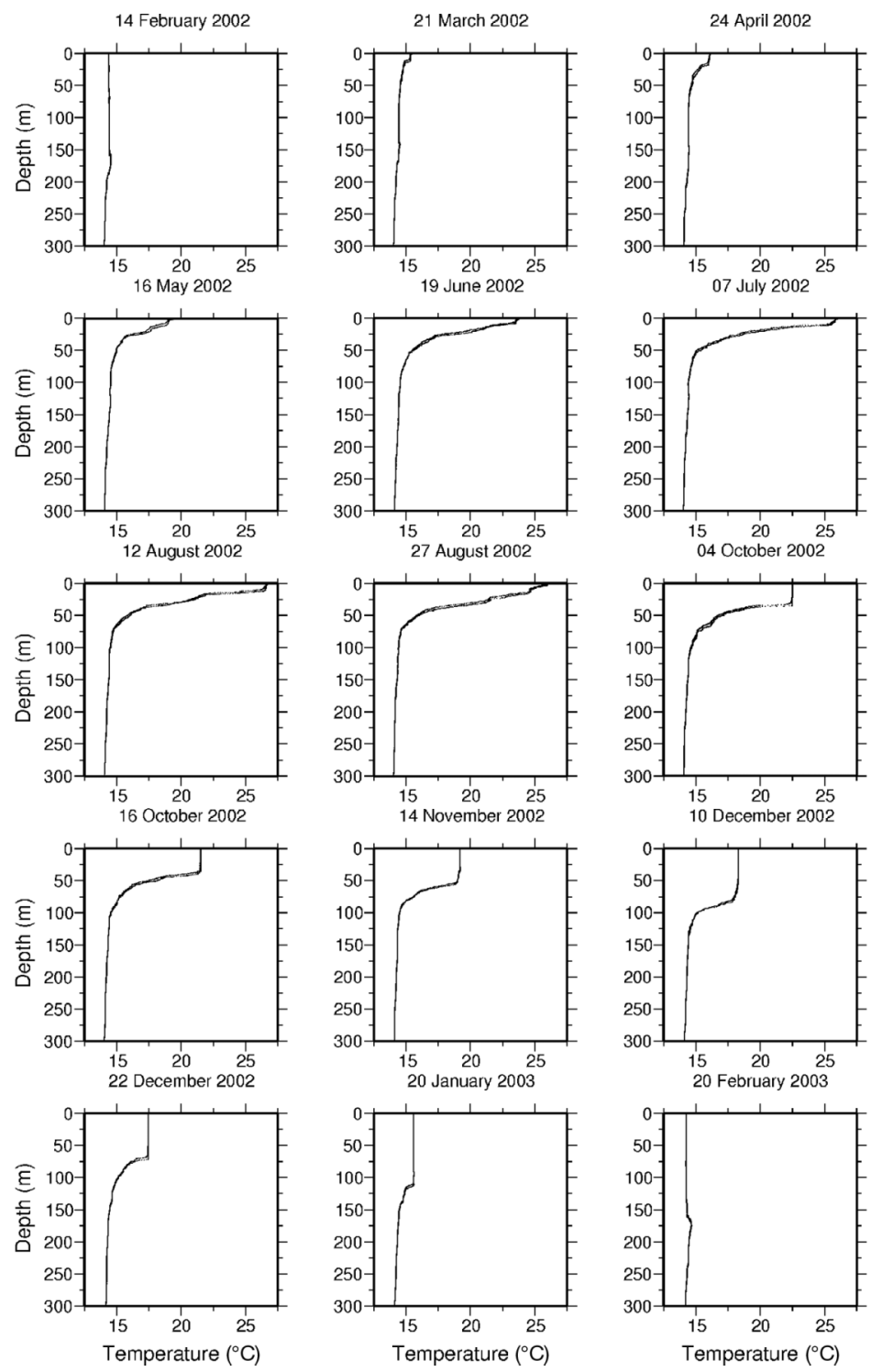

Fig. 3. Temperature profiles in the Gulf of Naples from the sea surface to the bottom, at $300 \mathrm{~m}$ in depth, measured across 15 different periods between February 2002 and February 2003. The main temperature variations are above $50 \mathrm{~m}$ depth. For depths greater than $160 \mathrm{~m}$ the seasonal temperature variation rarely reaches $0.1^{\circ} \mathrm{C}$.

ble variations in the sound velocity profiles, since the latter are less then $0.5 \mathrm{~m} / \mathrm{s}$ (that is $0.03 \%$ of the mean sound velocity in the seawater). Consequently, the rapid variations in salinity have not been considered in the construction of a sound velocity model of the Gulf of Naples.

Knowledge of the effects of the three physical parameters of temperature, pressure and 
salinity on the sound velocity profiles has allowed the construction of a multiparameter function of depth $z$ that simulates the sound velocity profiles throughout the year and for depths from 0-300 $\mathrm{m}$ in the Gulf of Naples, as- suming that the variations seen over the sampling period are stationary. The parameters of the function were obtained through the inversion of the 15 sound velocity profiles using the non-linear Simplex global optimization algo-
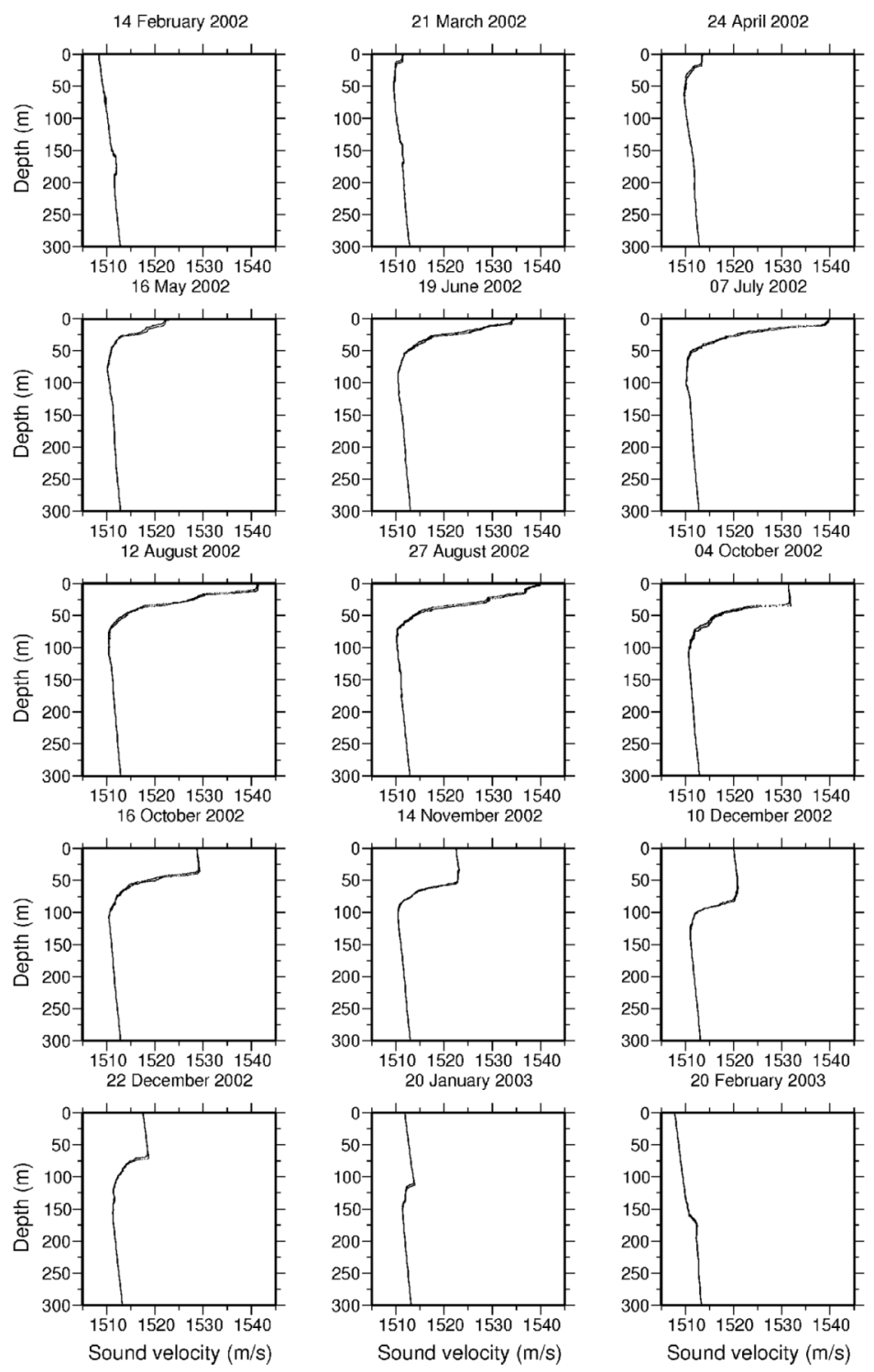

Fig. 4. Velocity profiles in the Gulf of Naples calculated from the salinity and temperature data obtained from the measurements carried out between February 2002 and February 2003. 
rithm (Nelder and Mead, 1965; Press et al., 1992) and creating a function of time for each parameter that interpolates the values obtained from the inversion. The chosen multiparameter model is described by the following function:

$$
V(z)=V_{0} /\left[1+\left(z / z_{c}\right)^{\gamma}\right]+\alpha+\beta z
$$

The linear dependence with depth $(\beta z)$ expresses the increase in the mechanical pressure. The parameter $\alpha$ represents the value of the sound velocity in sea water at a temperature of $15^{\circ} \mathrm{C}$ and a pressure of $1 \mathrm{~atm}$ (depth of $z=0 \mathrm{~m}$ ); the parameter $V_{0}$ represents the variation with respect to $\alpha$ in the sound velocity at the sea surface for a different temperature. The parameter $z_{c}$ gives information on the thickness of the surface isothermic layer. The adimensional parameter $\gamma$ amplifies the $z / z_{c}$ ratio and determines the negative slope of the function $V(z)$ for $z \approx z_{c}$.

\section{Noise sources in the underwater acoustic channel in the Gulf of Naples}

The noise signal of an underwater acoustic sensor is composed of electronic and acoustic noise, generated by the pressure fluctuations in the sea water due to the action of ambient sources (De Dominicis Rotondi, 1996).

Here, we consider only acoustic noise, and in particular we calculate the noise level around a frequency of $100 \mathrm{kHz}$ in the Gulf of Naples. Analytically, the Noise Level (NL) will be expressed in decibels $(\mathrm{dB})$ as the ratio between the noise pressure (or intensity) revealed with omnidirectional hydrophones and a reference pressure (or intensity) of a plane acoustic wave in the same frequency bandwidth. Typically, the reference pressure level used in underwater acoustics is $1 \mu \mathrm{Pa}$.

In the sea, there are various noise sources that are both artificial and natural, which have intensities that vary in time, space and frequency. The natural sources have physical origins (molecular thermal agitation, sea tides and turbulence), meteorological (wind, rain, hail, storms, wave backwash, glaciers), terrestrial (earthquakes, volcanoes, distant storms) and biological (cetaceans, crustaceans, fish); the artificial sources are due to human activities like seaside traffic, coastal industrial works, ports, boreholes for research into energy sources, and underwater acoustic communications besides those of interest.

This noise covers a vast frequency range that goes from frequencies lower than $1 \mathrm{~Hz}$ to frequencies higher than hundreds of $\mathrm{kHz}$. For the various frequency ranges, the trend of the spectrum shows particular characteristics as a consequence of the peculiarities of the various sources: each source has its own dominant frequency band in which it has a predominant aspect with respect to the other noise sources; sometimes, different sources can predominate in the same frequency band.

The range going from a few $\mathrm{kHz}$ to $100 \mathrm{kHz}$ is of interest in our study and is dominated by the noise generated by the impact on the sea surface of drops and water spray that arise from the breaking of the waves. A classical representation of the spectral distribution in the range from 100 $\mathrm{Hz}$ to $25 \mathrm{kHz}$ comes from the historic Knudsen curves (Knudsen et al., 1948), which came from a vast measurement campaign carried out during the Second World War by a group of researchers guided by V.O. Knudsen, along the coastal waters of various ocean regions. The curve, parameterized according to the condition of the sea or equivalently as a function of the wind velocity (in knots), shows a linear trend on a logarithmic frequency scale with a slope of around -20 $\mathrm{dB} / \mathrm{dec}$. A series of subsequent investigations in the Knudsen range, carried out by Wenz (1962) and Urick (1986), established a correlation between wind speed and the ambient noise spectrum level. Using the empirical formulae given by Coates (Coates, 1990; Stojanovic, 2006), it is possible to calculate the Noise Level (NL), from $1 \mathrm{~Hz}$ to $1 \mathrm{MHz}$, as a function of frequency (in $\mathrm{kHz}$ ), wind speed $w$ (in $\mathrm{m} / \mathrm{s}$ ) and shipping activity factor $s$, whose value ranges between 0 and 1 for low and high activity, respectively. From these formulae it is possible to infer that the shipping activity factor $\mathrm{s}$ is negligible at frequencies of about $100 \mathrm{kHz}$.

The calculation of the noise level of the Gulf of Naples for a frequency of $100 \mathrm{kHz}$ was carried out using Coates formulae and the mean wind velocity in the Gulf of Naples for both the summer and winter months. The wind velocities at 
$10 \mathrm{~m}$ above the sea surface were considered with the READY program starting from the FNL model (the model and program are available online on the website of the National Oceanic and Atmospheric Administration) they were sampled every six hours from February 2002 to February 2003 , the period in which the measurements of the physical parameters of the sea were taken. The mean wind velocity calculated for the summer months was $3.9 \mathrm{~m} / \mathrm{s}$, while for the winter months it was $5.6 \mathrm{~m} / \mathrm{s}$. The noise levels in the Gulf of Naples at the frequency of $f=100 \mathrm{kHz}$ are then $28 \mathrm{~dB} / \mu \mathrm{Pa}$ in summer and $30 \mathrm{~dB} / \mu \mathrm{Pa}$ in winter.

\section{Efficiency of the acoustic link for standard digital data transmission apparatus}

Knowledge of the physical parameters and the use of the function designed to simulate the sound velocity variations with depth and time in the Gulf of Naples has allowed us to carry out various simulations of high frequency acoustic propagation in the Gulf of Naples. Using eq. (2.1), a mean velocity profile was constructed for the summer and winter months, with the aim of studying the behavior of the acoustic propagation according to the variations in the sound velocity in the sea; the two velocity profiles are shown in fig. 5. We also calculated (fig. 5) the residuals for the summer and winter months as the difference between the values of the mean velocity profiles constructed using eq. (2.1) and those calculated using the profiles of fig. 4 . The residuals are less than $0.5 \mathrm{~m} / \mathrm{s}$ for both summer and winter mean velocity profiles, except for the first $50 \mathrm{~m}$ depth in summer profile where the residuals can reach a maximum value of $1.8 \mathrm{~m} / \mathrm{s}$ (only $0.1 \%$ of the sound velocity in the water). Below, we describe the calculation method used for the simulation of the signal propagation and for the calculation of the signal-to-noise ratio, considering a standard digital data transmission apparatus. This considers an omnidirectional transmitter that delivers sinusoidal acoustic signals for the transmission of a digital binary sequence, with a velocity of 2.4 $\mathrm{kbit} / \mathrm{s}$ at a dominant frequency of $100 \mathrm{kHz}$, in an
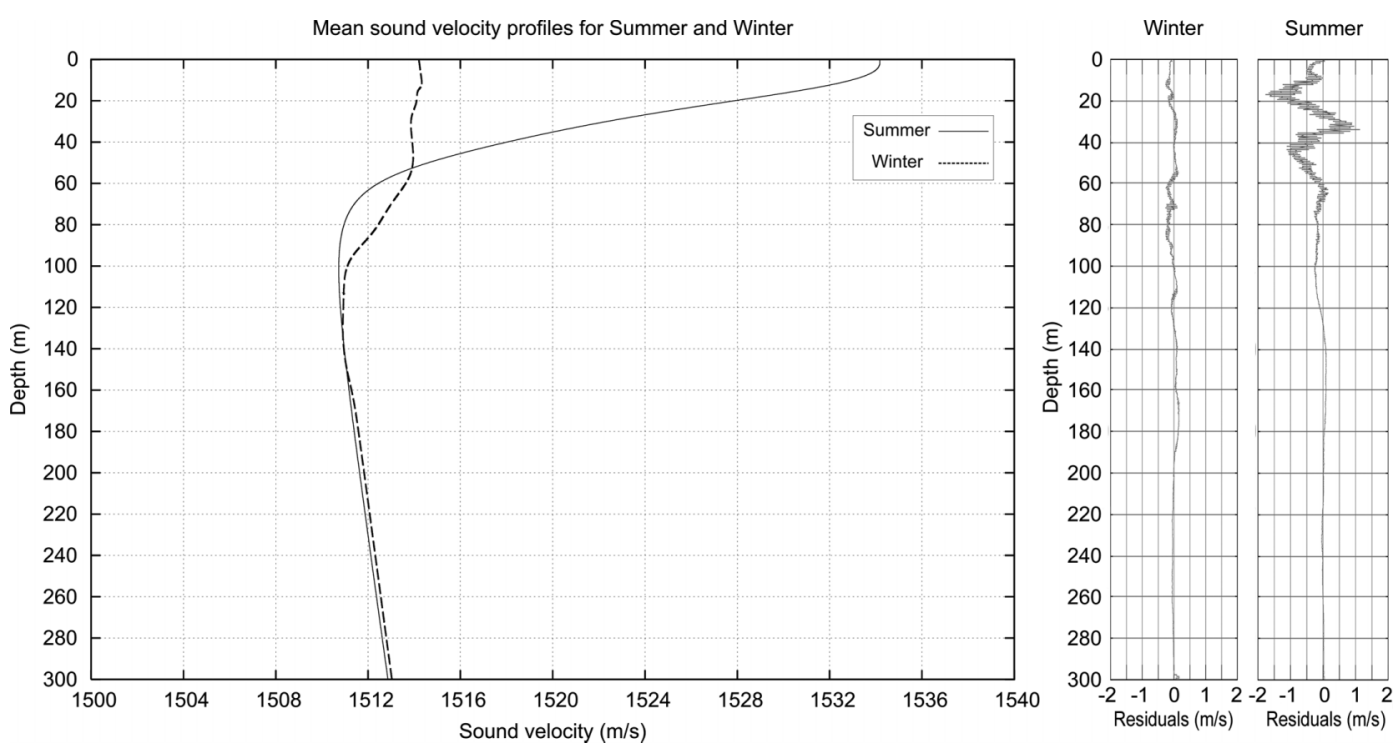

Fig. 5. Mean velocity profiles (left) for the summer and winter seasons calculated from eq. (2.1) and residuals (right) evaluated respect to the mean velocity profiles computed using the 15 velocity profiles obtained from data. Below $50 \mathrm{~m}$ depth the residuals are lesser than $0.5 \mathrm{~m} / \mathrm{s}$ for both summer and winter mean velocity profiles. 
operative band of $10 \mathrm{kHz}$. The power delivered to the antenna was assumed to be $0.4 \mathrm{~W}$ with a electroacoustic efficiency of $50 \%$.

\subsection{Description of the calculation method for simulation of signal propagation}

The propagation medium is described assuming a sea water layer (as previously described) and two solid layers (sea bottom sediments). The sediments samples taken from the first few meters of the sea bottom in the Gulf of Naples show that it is composed of clayey silt sediments in the first five meters and sand-siltclay sediments below, because of lower percentage of silt. From Hamilton studies on physical properties of marine sediments (Hamilton, $1978,1979,1980)$ we can therefore define a geoacoustic model of the seafloor assigning, for each solid layer, the thickness, the sound velocity ( $P$-waves), the $V p / V s$ ratio, and the density. The values of the physical parameters of the propagation medium used for the simulation are

Table I. Physical values assigned to the propagation medium for the simulation: the medium is described according to a liquid layer (sea water) and two solid layers (clayey silt sediments above sand-silt-clay sediments).

\begin{tabular}{ccccc}
\hline \hline $\begin{array}{c}\text { Stratum } \\
\text { typology }\end{array}$ & $\begin{array}{c}\text { Thickness } \\
(\mathrm{m})\end{array}$ & $\begin{array}{c}\text { Sound } \\
\text { velocity } \\
V p(\mathrm{~m} / \mathrm{s})\end{array}$ & $V p / V s$ & $\begin{array}{c}\text { Density } \\
\left(\mathrm{g} / \mathrm{cm}^{3}\right)\end{array}$ \\
\hline Sea water & 300 & $1510-1534$ & $\infty$ & 1.03 \\
Clayey silt & 5 & 1550 & $13.0 \pm 2$ & 1.40 \\
Sand-silt-clay & $\infty$ & 1650 & $9.4 \pm 2$ & 1.80 \\
\hline
\end{tabular}

given in table I. We also consider a variation of the $V p / V s$ ratio for both the solid layers to understand if it influences the propagation.

The discontinuity surfaces between the layers are flat and horizontal and no rough sea surface and seafloor are considered in this paper. Within the water layer, the sound velocity is assumed to vary continuously with depth, following the trend given in fig. 5. The model describing the characteristics of the propagation medium in the summer months essentially differs from the one relative to the winter months in terms of the sound velocity values in the water layer above the cut-off depth of about $50 \mathrm{~m}$. Table II gives the sound velocity values for the two models at increasing depths.

The simulations were carried out with the 3D dynamic ray-tracing code (Farra and Madariaga, 1987) with modifications to allow for the anelastic attenuation in the sea, which is considered by multiplying for each phase (direct $P$, multiple $P$ reflections in the water) the amplitude of the nonattenuated wave $A_{0}$ by a negative exponential

$$
A=A_{0} \exp (-\alpha x)
$$

where $\alpha$ is the attenuation coefficient and has the dimensions of inverse length and $x$ is the length of the ray-path.

From the empirical Thorp's formula (Thorp, 1967; Fisher and Simmons, 1977; Jensen et al., 2000)

$$
\begin{aligned}
& \alpha^{\prime}(\mathrm{dB} / \mathrm{km}) \approx 3.3 \cdot 10^{-3}+\frac{0.11 f^{2}}{1+f^{2}}+\frac{44 f^{2}}{4100+f^{2}}+ \\
& +3.3 \cdot 10^{-4} f^{2}
\end{aligned}
$$

the attenuation is expressed as a loss in decibels

Table II. Sound velocities of the summer and winter models for increasing depths. The main differences between the two models are above $50 \mathrm{~m}$ depth.

\begin{tabular}{lccccccccccc}
\hline \hline & & \multicolumn{1}{c}{ Depth $(\mathrm{m})$} & & & & & \\
& 0 & 10 & 20 & 30 & 40 & 50 & 60 & 80 & 100 & 160 & 300 \\
\hline Summer model $V p(\mathrm{~m} / \mathrm{s})$ & 1534 & 1533 & 1527 & 1523 & 1517 & 1514 & 1512 & 1511 & 1510 & 1511 & 1513 \\
Winter model $V p(\mathrm{~m} / \mathrm{s})$ & 1514 & 1514 & 1514 & 1514 & 1514 & 1514 & 1513 & 1512 & 1511 & 1511 & 1513 \\
\hline
\end{tabular}


per unit of distance as a function of the frequency $f$ (in $\mathrm{kHz}$ ) of the wave that propagates in the sea and it is indicated by $\alpha^{\prime}$ (Jensen et al., 2000), instead of $\alpha$. From the eq. (4.2) and considering a frequency of $100 \mathrm{kHz}$ we find that $\alpha^{\prime}$ is equal to $34.3 \mathrm{~dB} / \mathrm{km}$. Taking into consideration eq. (4.1), we can obtain $\alpha$ from $\alpha^{\prime}$ as follow:

$$
\begin{aligned}
& \alpha^{\prime} x=-20 \log \left(\frac{A}{A_{0}}\right) \approx 8.686 \alpha x \Rightarrow \\
& \Rightarrow \alpha \approx \frac{\alpha^{\prime}}{8.686}=3.95 \mathrm{~km}^{-1} .
\end{aligned}
$$

To study the reliability of the acoustic communication, we simulated the propagation from the transmitter to the receivers of a signal consisting of a sinusoidal transient of a $1 \mathrm{~s}$ duration and with a frequency of $100 \mathrm{kHz}$.

The transmitter, i.e. the source of the signal, is omnidirectional and was positioned at the three different depths of $1 \mathrm{~m}, 190 \mathrm{~m}$ and $299 \mathrm{~m}$ from the sea surface; for each transmitter position, the reception of the signal was simulated on a grid of $21 \times 12$ receivers, arranged in a vertical plane inside the area of interest showed in fig. 1 by a bright ellipse. The horizontal distance between the receivers is $\Delta x=100 \mathrm{~m}$ from the vertical axis through the source for up to 2 $\mathrm{km}$ in distance, while the vertical distance between the receivers is $\Delta z=25 \mathrm{~m}$, from $5 \mathrm{~m}$ in depth to $280 \mathrm{~m}$ in depth.

For each receiver, the particle displacement velocity $\boldsymbol{v}=\left(v_{x}, v_{y}, v_{z}\right)$ was simulated, from which it is possible to deduce the intensity of the signal, information that is useful for the determination of transmission losses. Indeed, the instantaneous acoustic intensity $I$ of field produced by force $F$ that acts normally on a unitary surface $A$ is $I=F v / A=p v$, where $p$ is the pressure and $v$ is the particle displacement velocity along the normal to the surface $A$. Considering that the time-scale of oceanographic changes is an order of magnitude greater than the time-scale of acous-tic propagation, it can be assumed that the density $\rho$ and the sound velocity $c$ are independent of time. With this assumption, both the pressure $p$ and the velocity potential $\phi(v=\nabla \phi)$ satisfy the wave equation, and using the linearized Euler equation (Jensen et al., 2000), this gives

$$
p=\rho c v \Rightarrow I=\rho c v^{2}
$$

where $v$ is the particle displacement velocity along the direction of acoustic wave propagation.

The simulated signal at each receiver is perturbed by the multipath effects, arising from the multiple reflections from the surface and the bottom of the sea. Because of this effect, with the transmission of digital data, the coding signals applied to the preceding bit are superimposed on the coding signals of the succeeding bit upon reception, causing an increase in the intersymbol interference.

\subsection{Calculation of the Signal-to-Noise Ratio (SNR)}

An object that radiates with a Source Level (SL) will be registered by a receiver with a lower Signal level (S) due to the Transmission Loss (TL) that it undergoes in its passage through the propagation medium. The Signal-to-Noise Ratio (SNR) for this receiver is obtained by subtracting the Noise Level (NL) from the Signal level (S), such that

$$
\operatorname{SNR}(d B)=S-N L=S L-T L-N L
$$

where all of the quantities under consideration are expressed in $\mathrm{dB}$.

To determine the limits beyond which the transmission is no longer reliable, using eq. (4.5) we can construct the SNR maps for a determined Source Level (SL) starting from the knowledge of the Transmission Loss (TL) at each receiver and the Noise Level (NL) for the Gulf of Naples. The Noise Level (NL) was discussed above in the second section, so we now consider the determination of the Source Level (SL) and calculate the Transmission Loss (TL) at the frequency of $100 \mathrm{kHz}$ on the whole grid of $21 \times 12$ receivers. The source level is determined according to the relationship (De Dominicis Rotondi, 1990)

$$
\operatorname{SNR}(\mathrm{dB})=170.5+10 \log (W e)+10 \log (\eta)+\mathrm{DI}
$$

where We represents the electrical power sup- 
plied to the antenna, $\eta$ the electroacoustic efficiency, and DI the Directivity Index. Considering a realistic example where the transmitter has an efficiency of $50 \%$ and the current supplied to the antenna is $185 \mathrm{~mA}$ with a tension of $3.24 \mathrm{~V}$, thus giving it an electrical power of 0.6 $\mathrm{W}$, from eq. (4.6) the source level will be $\mathrm{SL}=$ $=165 \mathrm{~dB}$. The transmission loss undergone by the signal at any specific receiver are obtained instead from the ratio between the mean intensity $I$ of the signal simulated at each receiver and the mean intensity $I_{0}$ of the signal simulated at one meter distance from the transmitter, giving the value in $\mathrm{dB}$ according to the relationship

$$
\mathrm{TL}(\mathrm{dB})=-10 \log \left(\frac{\langle I\rangle}{\left\langle I_{0}\right\rangle}\right)
$$

which, taking into account eq. (4.4), becomes

$$
\begin{aligned}
& \mathrm{TL}(\mathrm{dB})=-10 \log \left(\frac{\left\langle\boldsymbol{v}^{2}\right\rangle}{\left\langle\boldsymbol{v}_{0}^{2}\right\rangle}\right)= \\
& -10 \log \left(\frac{\left\langle\boldsymbol{v}_{x}^{2}+\boldsymbol{v}_{y}^{2}+\boldsymbol{v}_{z}^{2}\right\rangle}{\left\langle\boldsymbol{v}_{0 x}^{2}+\boldsymbol{v}_{0 y}^{2}+\boldsymbol{v}_{0 z}^{2}\right\rangle}\right) .
\end{aligned}
$$

Using eq. (4.8), it is therefore possible to calculate for each receiver the transmission loss knowing the mean value of the particle displacement velocity $\boldsymbol{v}=\left(v_{x}, v_{y}, v_{z}\right)$. The mean value is calculated across a period of time equal to the duration of the signal sent by the transmitter.

\section{Results obtained}

Starting from the knowledge of the transmission loss in the acoustic communication channel at the frequency of $100 \mathrm{kHz}$, and from the knowledge of the noise level in the Gulf of Naples, and considering a source with a $165 \mathrm{~dB}$ level, using eq. (4.5) we have constructed the maps that give the Signal-to-Noise Ratio (SNR) for the summer and winter months, for three different transmitter positions, considering both the multiple reflections and the direct phase only. If it is assumed that the band pass of the acoustic communication is $10 \mathrm{kHz}$ around the frequency of $100 \mathrm{kHz}$, and that the transmission velocity of the data from the emitter is 2.4 kbit/s, from Shannon's Capacity Theorem for a communication channel (Shannon, 1948) the signal-to-noise ratio must be greater than $22 \mathrm{~dB}$ for reliable information transmission.

Figures 6, 7, and 8 indicate in black the regions in the section of water in which the communication is not reliable, as in those zones of the channel in which the signal-to-noise ratio is less than $22 \mathrm{~dB}$. Where the transmission should be reliable ( $\mathrm{SNR}>22 \mathrm{~dB}$ ) a different color has been assigned to each SNR value calculated, in decreasing order from red to violet.

Figure 6 shows the signal-to-noise ratio for a transmitter positioned at $1 \mathrm{~m}$ in depth. A comparison between the summer and winter maps relative to the direct phase only shows that if the source is positioned at a low depth, the effect of the negative velocity gradient that is seen in summer does not favor surface communication for increasing distances. If, however, a comparison is made between the summer and winter maps that takes into account multiple reflections between the surface and the bottom, we see that the multipath effect predominates over the velocity gradient effect. Figures 7 and 8 show the signal-to-noise ratio for transmitters positioned at $190 \mathrm{~m}$ and $299 \mathrm{~m}$ in depth, respectively. From a comparison of the summer and winter maps, relative to the direct phase only, it can be seen that for these source depths the negative velocity gradient of the summer months no longer influences communication.

Comparing figs. 6,7 , and 8 for the cases in which multiple reflections are taken into account, it can be seen that if the source is a long way from the bottom and from the surface of the sea, the communication is less disturbed for up to 1.0 to $1.2 \mathrm{~km}$, because of the lesser influence of the surfaces that delimit the communication channel. Above this distance, the effects produced by the bottom and the free surfaces are similar, independently of the position of the source. The $V p / V s$ ratio does not influence the communication. In fact changing the $V p / V s$ ratio in the range illustrated in table I we obtain the same results as reported in figs. 6, 7, and 8 . Moreover, from these three figures (6, 7, and 8), it can be seen that the transmission is slightly favored in the summer period due to a lower noise 


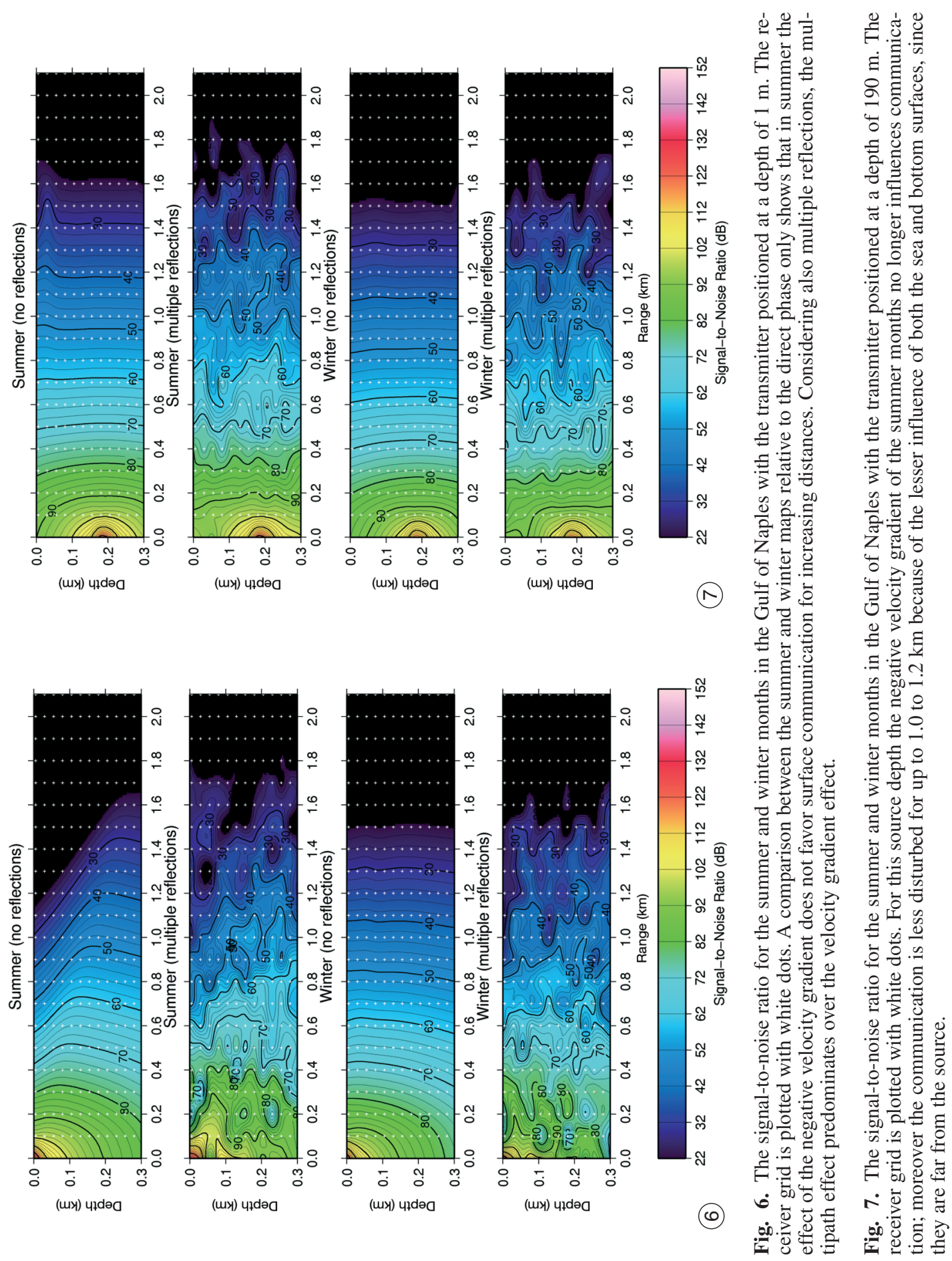



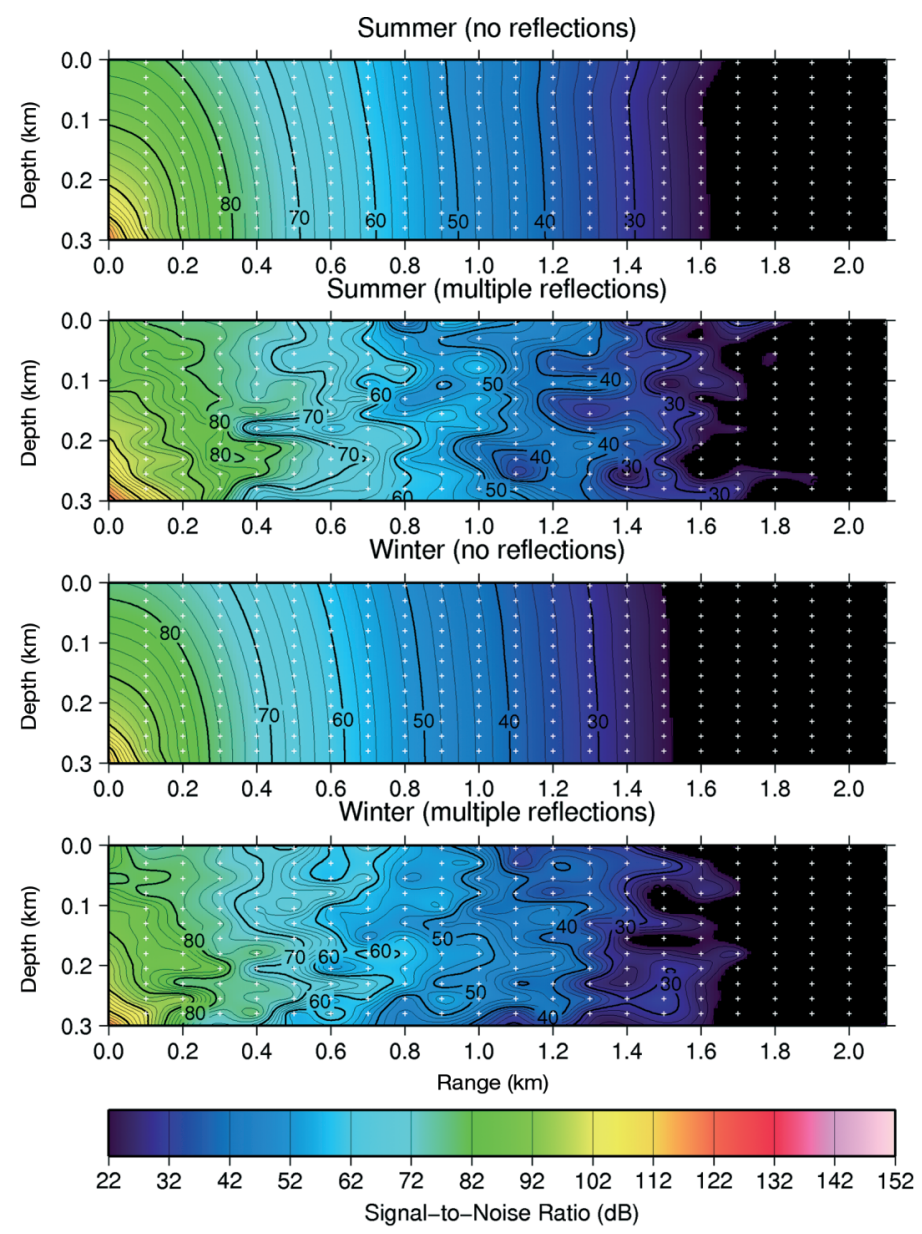

Fig. 8. The signal-to-noise ratio for the summer and winter months in the Gulf of Naples with the transmitter positioned at a depth of $299 \mathrm{~m}$. The receiver grid is plotted with white dots. For this source depth the negative velocity gradient of the summer months no longer influences communication, but the influence of the seafloor is magnified.

level from agitation of the sea. It is nevertheless important to bear in mind that on average the difference in the noise levels between the summer and winter months due to the conditions of the sea is only $4 \mathrm{~dB}$ in the Gulf of Naples.

Finally considering the information of fig. 5 we can see that below $50 \mathrm{~m}$ depth the mean sound velocity profiles for summer and winter season are similar, so also the SNR is stable during the year for transmitter-receiver connections below this depth.

\section{Conclusions}

Here, we have simulated the propagation of sinusoidal acoustic signals sent by an omnidirectional transmitter at the frequency of 100 $\mathrm{kHz}$ for the binary sequential digital transmission of information, in a propagation medium with the physical characteristics of the Gulf of Naples. Three different transmitter positions have been considered, at $1 \mathrm{~m}, 190 \mathrm{~m}$ and $299 \mathrm{~m}$ ( $1 \mathrm{~m}$ above the bottom) in depth. The reception 
of the transmitted signal was simulated, for all three cases, by a $21 \times 12$ receivers grid in the sea of $300 \mathrm{~m}$ in depth, for a range of $2 \mathrm{~km}$; from the simulation, the SNR maps have been constructed for all of the cases under consideration.

Considering a transmitter with a source level of $165 \mathrm{~dB}$ and a velocity of $2.4 \mathrm{kbit} / \mathrm{s}$, for a band pass of $10 \mathrm{kHz}$, it is seen that the transmission of information is reliable up to distances of 1400-1600 m between source and receiver below $50 \mathrm{~m}$ depth and in all seasons of the year, because the mean sound velocity profiles for summer and winter months are similar below this depth. The summer seasonal effects on the sound velocity cannot be overlooked when the transmitter is positioned at the surface or at a low depth. The presence of the sea surface and bottom produce, moreover, important multipath effects that result in errors in the decoding process of the bit sequence of the transmitted information, particularly if the transmitter is positioned near the surfaces limiting the acoustic channel. Although the sea surface and bottom produce multipath effects, the $V p / V s$ ratio of the sedimentary layers does not influence the communication.

If the transmitter is supplied with directionality, the source level can then be increased up to $40 \mathrm{~dB}$, and in this case it is possible to reach a distance of $2000 \mathrm{~m}$ while minimizing the multipath effects due to the multiple reflections. Figures 6, 7, and 8 that are relative to the direct phase only give indications as to the SNR that would be present in the case in which the transmitter is directional; these values must then be increased by the factor of directionality (DI) of the source, which in the case examined in this article is equal to zero, because it involves a non-directional source.

This study represents a feasibility test of using the acoustic communication to transmit data acquired on the seafloor of the Gulf of Naples. In this region a complex geophysical monitoring system operates for the surveillance of the Neapolitan volcanoes (Mt.Vesuvius and Campi Flegrei caldera). All the instruments are installed inland, while on the seafloor only temporary experiments have been carried out. This study offers a new perspective on the future developments of the monitoring system by the in- stallation of geophysical stations on the seafloor with data transmission by acoustic link to a moored buoy that provides connectivity back to the land by a radio link.

\section{Acknowledgements}

The authors would like to thank the engineers of the Whitehead Alenia Sistemi Subacquei SpA of Monteruscello (NA) for providing the technical specifications of the underwater acoustic transmitters and of the modalities of the underwater acoustic transmission of digital data. We also thank Dr. M. Ribera for providing us with the data on the physical characteristics of the water channel in the Gulf of Naples after the measurements carried out by A. Dohrn Zoological Station staff in the period February 2002-February 2003.

This work was funded by MIUR (Ministry of Education, University and Research) PONSisMa project.

\section{REFERENCES}

Adams, A., O. Hinton, B. Sharif, G. Salles, N. OrR and C. Tsiminedes (2000): An experiment in sub-sea networks - The LOTUS sea trials, in Proceedings of the ECUA'00, Lyon, France.

AKYILDiZ, I.F., D. Pompili and T. Melodia (2005): Underwater acoustic sensor networks: research challenges, Ad Hoc Networks, 3 (3), 257-279.

Badia, L., M. Mastrogiovanni, C. Petrioli, S. Stefanakos and M. ZORZI (2006): An optimization framework for joint sensor deployment, link scheduling and routing in underwater sensor networks, in Proceedings of the First ACM International Workshop on Underwater Networks (WUWNet'06), Los Angeles, California, U.S.A., 56-63.

Baggeroer, A. (1984): Acoustic telemetry - An overview, IEEE J. Ocean. Eng., 9, 229-235.

Benson, B., G. Chang, D. Manov, B. Graham and R. KASTNER (2006): Design of a low-cost acoustic modem for moored oceanographic applications, in Proceedings of the First ACM International Workshop on Underwater Networks (WUWNet'06), Los Angeles, California, U.S.A., 71-78.

CATIPOVIC, J.A. (1990): Performance limitations in underwater acoustic telemetry, IEEE J. Ocean. Eng., 15, 205-216.

Cazzolato, B.S., P. Nelson, P. Joseph and R.J. Brind (2001): Numerical simulation of optimal deconvolution in a shallow-water environment, J. Acoust. Soc. Am., 110, 170-185.

Chen, C.T. and F.J. Millero (1977): Speed of sound in seawater at high pressure, J. Acoust. Soc. Am., 62, 11291135 . 
COATES, R.F.W. (1990): Underwater Acoustic Systems (John Wiley \& Sons Ltd., New York), pp. 188.

COATES, R.F.W. (1993): Underwater acoustic communications, in Proceeding of OCEANS '93, Victoria, Canada, III.420-III.425.

Coppens, A.B. (1981): Simple equations for the speed of sound in Neptunian waters, J. Acoust. Soc. Am., 69, 862-863.

DAHL, P.H. (2004): The sea surface bounce channel. Bubble-medianted energy loss and time/angle spreading, in High Frequency Ocean Acoustics, edited by $\mathrm{M}$. PORTER, M. Siderius and W. KUPERMAN (American Institute of Physics), 194-203.

De Dominicis Rotond, A. (1990): Principi di Elettroacustica Subacquea (Ed. ELSAG, Genova), vol. I, pp. 422.

De Dominicis Rotondi, A. (1995): Principi di Elettroacustica Subacquea. Le Caratterizzazioni dell'Smbiente Operativo Marino (Edizioni Scriba), vol. II, pp. 256.

De Dominicis Rotond, A. (1996): Principi di Elettroacustica Subacquea. Il Canale Acustico Marino (Edizioni Scriba), vol. III, pp. 512.

Del Grosso, V.A. (1974): New equation for the speed of sound in natural waters (with comparisons to other equations), J. Acoust. Soc. Am., 56, 1084-1091.

FARRA, V. and R. MADARIAGA (1987): Seismic waveform modeling in heterogeneous media by ray perturbation theory, J. Geophys. Res., 92, 2697-2712.

FISHER, F.H. and V.P. SimMONS (1977): Sound absorption in sea water, J. Acoust. Soc. Am., 62, 558-564.

Hamilton, E.L. (1978): Sound velocity gradients in marine sediments, J. Acoust. Soc. Am., 65, 909-922.

Hamilton, E. L. (1979): Vp/Vs and Poisson's ratios in marine sediments and rocks, J. Acoust. Soc. Am., 66, 1093-1101.

Hamilton, E.L. (1980): Geoacoustic modeling of the sea floor, J. Acoust. Soc. Am., 68, 1313-1340.

JAFFE, J. and C. SCHURGERS (2006): Sensor networks of freely drifting autonomous underwater explorers, in Proceedings of the First ACM International Workshop on Underwater Networks (WUWNet'06), Los Angeles, California, U.S.A., 93-96.

Jensen, F.B., W.A. Kuperman, M.B. Porter and H. Schmidt (2000): Computational Ocean Acoustics (Springer-Verlag), pp. 600.

Kinsler, L.E., A.R. Frey, A.B. Coppens and J.V. SAnders (2000): Fundamentals of Acoustics (John Wiley \& Sons Ldt., New York), 4th edition, pp. 560.

Knudsen, V.O., R.S. Alford and J.W. EMLing (1948): Underwater ambient noise, J. Mar. Res., 22, 410-429.

MacKenzIE, K.V. (1981): Nine-term equation for the sound speed in the oceans, J. Acoust. Soc. Am., 70, 807-812.

Nelder, J.A. and R. MEAD (1965): A simplex method for function minimization, Comput. J., 7, 308-313.

PompILI, D., T. MElodia and I.F. Akyildiz (2006): Deployment analysis in underwater acoustic wireless sensor networks, in Proceedings of the First ACM Internation- al Workshop on Underwater Networks (WUWNet'06), Los Angeles, California, U.S.A., 48-55.

Preisig, J. (2006): Acoustic propagation considerations for underwater acoustic communications network development, in Proceedings of the First ACM International Workshop on Underwater Networks (WUWNet'06), Los Angeles, California, U.S.A., 1-5.

Press, W.H., S.A. Teukolsky, W.T. Vetterling and B.P. FLANNERY (2003): Numerical Recipes in Fortran 77: the Art of Scientific Computing (Cambridge University Press), 2nd edition, pp. 992 (available online at: <http:// www.nr.com>).

SHANNON, C.E. (1948): A mathematical theory of communication, Bell System Technical J., 27, 379-423, 623-656.

Smith, K.B., A.A.M. Abrantes and A. LARraZA (2003): Examination of time-reversal acoustics in shallow water and applications to noncoherent underwater communications, J. Acoust. Soc. Am., 113, 3095-3110.

Sozer, EM., M. Stojanovic and J. G. Proakis (2000): Underwater acoustic networks, IEEE J. Ocean. Eng., 25, 72-83.

StoJANOvic, M. (1996): Recent advances in high-speed underwater acoustic communications, IEEE J. Ocean. Eng., 21, 125-136.

StojanOvic, M. (2004): Refocusing techniques for high rate acoustic communications, J. Acoust. Soc. Am., 117, 1173-1185.

STOJANOVIC, M. (2006): On the relationship between capacity and distance in an underwater acoustic communication channel, in Proceedings of the First ACM International Workshop on Underwater Networks (WUWNet'06), Los Angeles, California, U.S.A., 41-47.

Stojanovic, M., J.A. Catipovic and J.G. Proakis (1993): Adaptive multichannel combining and equalization for underwater acoustic communications, J. Acoust. Soc. Am., 94, 1621-1631.

Stojanovic, M., J.A. Catipovic and J.G. Proakis (1994): Phase coherent digital communications for underwater acoustic channels, IEEE J. Ocean. Eng., 19, 100-111.

Stojanovic, M., J.A. Catipovic and J.G. Proakis (1995): Reduced complexity spatial and temporal processing of underwater acoustic communication signals, $J$. Acoust. Soc. Am., 98, 961-672.

THORP, W.H. (1967): Analytic description of the low-frequency attenuation coefficient, J. Acoust. Soc. Am., 42, p. 270.

UricK, R.J. (1986): Ambient Noise in the Sea (Peninsula Publishing), pp. 205.

WENZ, G.M. (1962): Acoustic ambient noise in the ocean: spectra and sources, J. Acoust. Soc. Am., 34, 1936-1956.

WoNG, G.S.K. and S. ZHU (1995): Speed of sound in seawater as a function of salinity, temperature and pressure, J. Acoust. Soc. Am., 97, 1732-1736.

(received December 20, 2006; accepted September 20, 2007) 